\title{
Perfilando los consumidores de cannabis que autocultivan a pequeña escala
}

\author{
Manuel Isorna ${ }^{1 *}$, Bárbara G. Amado ${ }^{2}$, Berta Cajal $^{3}$ y Dolores Seijo ${ }^{2}$ \\ ${ }^{1}$ Facultad Ciencias de la Educación y Deporte, Universidad de Vigo (España). \\ ${ }^{2}$ Departamento de Psicología Organizacional, Juridico-Forense y Metodología de las Ciencias del Comportamiento, Universidad de Santiago de Compostela (España). \\ ${ }^{3}$ Facultad de Psicología, Universidad de las Islas Baleares (España).
}

\begin{abstract}
Resumen: El cannabis es la sustancia ilícita más consumida a nivel mundial, con una prevalencia estimada entre el 2.8 y el $5.0 \%$ de la población adulta. El auge del consumo de cannabis ha llevado a un aumento en el cultivo para el propio consumo. Como consecuencia, nos planteamos un estudio de campo con el objetivo de contrastar si consumidores con y sin autocultivo se diferencian en el perfil sociodemográfico, patrones de consumo, riesgo de consumo problemático, dependencia, y síntomas y trastornos asociados. Para ello, 761 consumidores de cannabis, 480 hombres y 281 mujeres, de los cuales 251 autocultivaban para su consumo (182 hombres y 69 mujeres), con edades comprendidas entre los 18 y 60 años $(M=26.36$, $D T=5.68)$, respondieron a un cuestionario sociodemográfico y de patrones de consumo, a escalas de medida del consumo problemático de cannabis, dependencia del cannabis, abuso del cannabis, y de dependencia de la nicotina y del alcohol. Los resultados mostraron que el autocultivo es característico mayoritariamente de los varones, con bajo nivel académico y laboralmente activos. Asimismo, la práctica del autocultivo se relaciona con un mayor consumo de cannabis, una mayor implicación en la venta ilegal del producto, policonsumo y con problemáticas psicosociales derivadas de dicho consumo. Adicionalmente, los que autocultivan informan de mayores niveles de dependencia y de riesgo de consumo problemático de cannabis. Se discuten las implicaciones para la prevención y tratamiento de los trastornos por consumo de cannabis.
\end{abstract}

Palabras clave: autocultivo; cannabis; consumo; dependencia; consumo problemático.

\section{Introducción}

Los preparados del cannabis sativa (i.e., hachís, marihuana) constituyen la droga de uso ilegal más consumida a nivel mundial con una prevalencia estimada entre el 2.8 y el $5.0 \%$ de la población adulta (United Nations Office on Drugs and Crime, 2013). En el caso concreto de España estas tasas serían muy superiores. Así, la encuesta EDADES de 2011 del Observatorio Español sobre Drogas (OED, 2013) puso al descubierto que el $14.1 \%$ de la población entre los 15 y 34 años había fumado cannabis en el último mes, en tanto la encuesta ESTUDES de 2012-2013 estableció que cursa de inicio aproximadamente a los 15 años, más en los varones y una prevalencia del $26.6 \%$ entre los jóvenes de 14 a 18 años (Observatorio Español sobre Drogas, 2014), adaptándose los datos a una trayectoria de escalada en estas variables.

El consumo en exceso de cualquier droga provoca un trastorno clínico, recogido en el DSM-V (American Psychiatric Association, 2014) bajo la categoría diagnóstica de Trastorno por Consumo de Cannabis cuando la droga de uso es ésta, y en la CIE-10 en la categoría Trastornos mentales y del comportamiento debidos al consumo de cannabinoides (Organización Mundial de la Salud, 1992). La prevalencia de ca-

* Dirección para correspondencia [Correspondence address]:

Manuel Isorna Folgar. Facultad Ciencias Educación y Deporte. Campus

A Xunqueira s/n, 36005 Pontevedra (España).

E-mail: isorna.catoira@uvigo.es
Title: Profiling small scale domestic grower cannabis users.

Abstract: Cannabis is the most widely used worldwide drug with prevalence between 2.8 and $5.0 \%$ of the adult population. Increasing use of cannabis has carried out to an escalation in home growing for selfconsumption. As a consequence, a field study to contrast if the domestic cannabis growers exhibited a different sociodemographic profile, a consumption pattern, a problematic use risk, dependence level, as well as on the clinical symptoms and related disorders, was designed. As for this, 761 cannabis users, 480 males and 281 females, of whom 251 were homegrowers for personal use (182 males and 69 females), with ages ranging from 18 to 60 years $(M=26.36, S D=5.68)$, answered to a sociodemohgraphic and consumption pattern questionnaire, and to cannabis problematic use, cannabis dependence, cannabis abuse, and nicotine and alcohol dependence scales. The results showed that homegrowers are mostly males, with low academic training and working. Moreover, homegrowers have a higher cannabis consumption rates, are more implied in cannabis selling, polysubstance use, and with more psychosocial problem driven from cannabis use. Finally, homegrowers inform of higher problematic cannabis use and a higher level of cannabis dependence. The implications of the results for prevention police, and treatment of the cannabis use disorder are discussed.

Key words: homegrowing; cannabis; use; dependence; problematic use.

sos clínicos de consumo ha sido cifrada por la American Psychiatric Association en la población norteamericana en aproximadamente el $3.4 \%$ en jóvenes entre 12 y 17 años y en el $1.5 \%$ en mayores de 18 años, siendo la tasa observada menor en mujeres y decreciendo con la edad. Asimismo, la American Psychiatric Association también informa de importantes diferencias en la prevalencia mediadas por la raza, etnia y cultura. Dado que las tasas de consumo de cannabis en la población española son muy superiores y que el mejor predictor del trastorno por consumo de cannabis es el consumo habitual de esta sustancia, se espera que la prevalencia de trastornos clínicos también lo sea. Así se desprende que el $18.1 \%$ de las admisiones a tratamiento por drogas fueron para el tratamiento del trastorno por consumo de cannabis, siendo casi exclusivo para los menores de 18 años, el 86.4\% (Observatorio Español sobre Drogas, 2011).

Estamos, pues, ante un problema de salud muy reseñable que afecta a la población en general y, muy especialmente, a los jóvenes por lo que se prevé una evolución ascendente. Adicionalmente el consumo de cannabis y el trastorno por consumo de cannabis traen aparejados (comorbilidad, diagnóstico dual) disfunciones, síntomas y trastornos como la depresión, ansiedad, síntomas psicóticos, personalidad antisocial, déficits en las funciones cognoscitivas, o consumo (o trastornos por el consumo) de otras sustancias (American Psychiatric Association, 2014; Bovasso, 2001; Davis, Compton, Wang, Levin, y Blanco, 2013; Isorna, Fernández-Ríos, 
y Souto, 2010; Maldonado, Valverde, y Berrendero, 2006; Patton et al., 2002; Verdejo-García, 2011).

Este estado de la cuestión ha conllevado que se iniciara y extendiera en los países occidentales el cultivo ilícito de plantas de cannabis (International Narcotics Control Board, 2011). El auge de cultivo se relaciona, principalmente, con el autocultivo a pequeña escala (en adelante autocultivo) que se estima lo practican alrededor del $40 \%$ de los consumidores (Gamella y Jiménez, 2003). Igualmente, en la última década se ha producido un aumento en la potencia del cannabis (THC) (Potter, 2014; Potter, Clark, y Brown, 2008) como consecuencia de modificaciones genéticas de la planta y del uso de instrumentos para el cultivo más sofisticados, especialmente en cultivos de interior, lo que conlleva a un incremento en el desarrollo de un trastorno por consumo de cannabis y mayores daños a la salud (Di Forti et al., 2014; Hall y Degenhardt, 2009). El autocultivo a pequeña escala facilita la disponibilidad de la sustancia, un abaratamiento del coste y, por ende, la extensión del consumo (Coffey, Lynskey, Wolfe, y Patton, 2000; Gervilla y Palmer, 2010; von Sydow, Lieb, Pfister, Höfler, y Wittchen, 2002).

Como consecuencia de todo ello nos planteamos un estudio de campo con el objeto de contrastar si el autocultivo participa de unos efectos diferenciales en el perfil sociodemográfico, los patrones de consumo, riesgo de consumo problemático, dependencia y síntomas y trastornos asociados, en comparación con consumidores que no practican el autocultivo.

\section{Método}

\section{Participantes}

Participaron en el estudio 761 consumidores de cannabis (480 hombres y 281 mujeres), de los cuales 251 autocultivaban cannabis para su consumo (182 hombres y 69 mujeres). Las edades de los participantes oscilaban entre los 18 y los 60 años $(M=26.36, D T=5.68)$.

\section{Procedimiento y diseño}

Se diseñó un estudio cuasi-experimental de campo, realizado entre junio de 2012 y febrero de 2014. Se procedió con un muestreo no probabilístico y para la selección de los participantes se siguió un sistema de bola de nieve: a partir de un primer contacto con consumidores de cannabis éstos actuaban como informadores para identificar a otros consumidores. Los cuestionarios y escalas fueron administrados por investigadores previamente entrenados; ciegos a los objetivos del estudio; en sesiones de aplicación individuales y en condiciones de no-abstinencia. El tiempo de administración osciló entre los 15 y los 30 minutos. El criterio para la selección fue el auto-etiquetaje como consumidor habitual de cannabis (Martín del Moral y Lorenzo, 1998). Se siguió el mismo criterio de selección (auto-etiquetaje) para la diferenciación entre participantes que autocultivaban y los que no lo hacían. La participación fue voluntaria, prestando consentimiento informado, respetando los principios éticos de la Declaración de Helsinki (2013) y de la Ley de Protección de Datos 15/1999.

Sometida a análisis la sensibilidad del diseño y dado que el tamaño muestral varía de unos análisis a otros, encontramos que para la comparación de medias con la prueba $t$ para muestras independientes, la probabilidad de detección $(1-\beta)$ de diferencias significativas $(\alpha<.05)$ y para un tamaño del efecto medio, oscilaba entre el $99 \%$ y el $100 \%$; para la comparación de la media de la población con un valor de prueba del 100\%; para los resultados de las pruebas de ji cuadrado aplicadas entre el 99\% y el 100\%; y para el MANOVA ejecutado del 100\%. En consecuencia, el diseño ejecutado es altamente sensible a la detección de diferencias significativas.

\section{Instrumentos de medida}

Para alcanzar los objetivos planteados, se aplicaron los siguientes instrumentos de medida:

Cuestionario sociodemográfico. Se creó un cuestionario sociodemográfico ad hoc (i.e., sexo, edad, nivel de estudios [certificado escolar, graduado escolar, medio y universitario], con quien vive [solo o en vivienda compartida ya sea con familiares o amigos], ocupación [inactivo, activo y estudiante]) y de patrones de consumo (i.e., con/sin autocultivo, dónde adquieren las semillas o instrumentos para el cultivo [Grow Shops o Internet], frecuencia de consumo de marihuana en los últimos treinta días [ninguno, entre 1 y 3 días, entre 4 y 9 días, más de 10 días, todos o casi todos los días], consumo de cocaína o drogas sintéticas en algún momento de su vida, lugar habitual en el que adquieren el cannabis [lugar público o vivienda], qué drogas consumen al salir de noche [la combinación de cannabis y otras drogas legales o cannabis junto a otras drogas ilegales], han vendido drogas y cuál era el motivo principal para la venta [ganar dinero o consumir gratis], preocupación por el dinero invertido en cannabis y la necesidad de poner excusas sobre su gasto, fumar más en solitario y relacionarse en mayor medida con gente que también consume).

Cannabis Problems Questionnaire (CPQ) (Copeland, Gilmour, Gates, y Swift, 2005). Este instrumento, que consta de 22 ítems de respuesta dicotómica (sí vs. no) y que evalúa los problemas psicosociales relacionados con el consumo habitual de cannabis, fue traducido al castellano por los investigadores y sometido a un procedimiento de back-translation. La medida se compone de tres dimensiones (Copeland et al., 2005): consecuencias físicas $(\alpha=.78)$, psicológicas $(\alpha=.71)$ y sociales $(\alpha=.55)$ del consumo de cannabis. Con los participantes en el estudio hemos obtenido una $\alpha$ de .82 .

Cannabis Abuse Screening Test (CAST) (Legleye, Karila, Beck, y Reynaud, 2007). Escala de 6 ítems cuyo objeto es la detección del consumo problemático de cannabis o patrones de abuso mediante la evaluación de las dificultades para controlar el consumo y sus consecuencias negativas sobre la salud o las relaciones sociales en el último año. Los ítems se 
contestan en una escala de 5 puntos $(0=$ Nunca, $1=$ Raramente, $2=$ De vez en cuando, $3=$ Bastante a menudo y $4=$ Muy a menudo). La escala mostró, con los participantes en el estudio, ser consistente internamente $(\alpha=.76)$.

Severity of Dependence Scale (SDS) (Gossop et al., 1995). La SDS, de la que se tomó la adaptación castellana para el consumo de cannabis de González-Saiz, de las Cuevas, Barrio, y Domingo-Salvany (2008), evalúa los aspectos psicológicos de la dependencia o consumo compulsivo. Se compone de 5 ítems a los que los individuos responden en una escala tipo likert (items 1 a 4: $0=$ Nunca $/$ casi nunca, $1=$ Algunas veces, $2=$ A menudo, 3 = Siempre/casi siempre; ítem 5: $0=$ Nada difícil, $1=$ Bastante difícil, $2=$ Muy difícil, $3=$ Imposible). Proporciona una puntuación total de dependencia con un rango que va desde 0 (sin dependencia alguna) a 15 (máximo nivel de dependencia). La fiabilidad obtenida a partir de los datos de nuestra muestra fue de $\alpha=.76$.

Alcohol Use Disorders Identification Test [Cuestionario de Identificación de los Trastornos Debido al Consumo de Alcohol] (AUDIT) (Saunders, Aasland, Babor, de la Fuente, y Grant, 1993), tomando la adaptación española de RubioValladolid, Bermejo-Vicedo, Caballero Sánchez-Serrano, y Santo-Domingo Carrasco (1998). Este test identifica a las personas con un patrón de consumo perjudicial o de riesgo de alcohol. Está compuesto por 10 ítems sobre el consumo reciente, síntomas debidos a la dependencia y problemas relacionados con la ingesta de alcohol. La escala de repuesta tipo Likert consta de 3 o 5 alternativas. Sometida a contraste la fiabilidad con los participantes en el estudio obtuvimos una $\alpha$ de .76.

Fagerström Test for Nicotine Dependence (FTND) (Heatherton, Kozlowski, Frecker, y Fagerström, 1991) para la medida de la dependencia de la nicotina. Consta de 6 ítems (2 ítems: respuesta tipo likert con 4 alternativas de respuesta; 4 ítems: escala dicotómica de respuesta). Una puntuación igual o superior a 6 es indicativa de dependencia de la nicotina. A nivel de clasificación, una puntuación entre 0 y 4 clasifica la dependencia como de nivel bajo; de 5 moderado, y $\geq 6$ alto. Con los participantes en este estudio obtuvimos una $\alpha$ de .67 .

\section{Análisis de datos}

Los estudios de comparación de medias son muy útiles en la investigación científica, pero sus resultados presentan dificultades de transferencia a la práctica profesional al no poder generalizarse los mismos a casos $(N=1)$. Por ello, se recomienda la combinación de ambos tipos de análisis (American Psychiatric Association, 2014; Arce, Fariña, Carballal, y Novo, 2006; Palmer, Borrás, Pérez-Pareja, Sesé, y Vilariño, 2013).

La asociación entre variables categóricas se abordó por medio del estadístico ji cuadrado obteniendo el tamaño del efecto para tablas $2 \times 2$ con phi y para tablas diferentes a $2 \times$ 2 con la phi de Cramer. La distribución de contingencias en una variable también fue analizada por el estadístico ji cua- drado siendo, en este caso, el tamaño del efecto computado por $\delta$ (Hedges y Olkin 1985), derivado del procedimiento de Kraemer y Andrews, 1982), equivalente a la $b$ de Cohen. Los contrastes a posteriori se ejecutaron con el nivel de protección de bonferroni. El contraste de una proporción observada con un valor dado o de prueba se llevó a cabo por medio de la prueba $Z$, obteniéndose el tamaño del efecto también por medio de la $\delta$ de Hedges y Olkin. La comparación de medias de dos grupos se abordó con la prueba $t$ de student para dos grupos independientes, y la comparación de una media con un valor dado o de prueba también con el estadístico $t$ de student para una muestra, calculándose para la estimación del tamaño del efecto la $d$ de Cohen (1988). Finalmente, la comparación de medias en un grupo de variables relacionadas teórica y estadísticamente (i.e., correlacionadas) fue llevada a cabo con un MANOVA, derivándose el tamaño del efecto de $\eta^{2}$ y la región de normalidad de la siguiente ecuación: $M_{\text {normativa }}-1.96 * D T_{\text {normativa }}<M_{\text {observada }}<M_{\text {normativa }}$ $+1.96 * D T_{\text {normativa. }}$ Los tamaños del efecto se interpretaron conforme a las categorías de Cohen (1988).

\section{Resultados}

\section{Respecto a variables sociodemográficas}

Los resultados mostraron que el autocultivo (.33) no es una característica significativa, y con un tamaño del efecto grande, de los consumidores de cannabis, $\chi^{2}(1, N=761)=$ $88.14, p<.001, \delta=-0.88$. No obstante, la prevalencia de consumidores de cannabis (comparación entre la contingencia observada, con el criterio de significatividad estadística, esto es, .05 que sería el margen de error admisible o contingencias despreciables) que autocultivan es significativa y con un tamaño del efecto grande, $Z(N=761)=11.16, p<.001$, $\delta=0.88$, autocultivando significativamente más, $\chi^{2}(1, N=$ $761)=14.32, p<.001, d=0.28$, los varones (.38) que las mujeres (.25). La adquisición de los productos para el autocultivo se formaliza general y exclusivamente en tiendas Grow Shops (.64). Por su parte, el control paterno, que se asocia a vivir en el hogar paterno y contrariamente a lo esperado (Becoña et al., 2013), es independiente del autocultivo, $\chi^{2}(1$, $N=745)=0.33, n s, d=-0.04$.

Los resultados del estudio de la asociación entre autocultivo (sí vs. no) y el título académico alcanzado (certificado escolar, graduado escolar, medio y universitario) evidenciaron diferencias significativas, $\chi^{2}(3, N=748)=12.31, p<.01, \phi^{\prime}$ de Cramer $=.13$. Los análisis post hoc pusieron de manifiesto $(p=.05 / 6=.008)$ que los que obtuvieron un título universitario (.27) autocultivan significativamente menos, con un tamaño del efecto bajo $(d=0.36)$, que los que tienen la certificación de escolaridad (.48).

Para la ocupación (inactivo, activo y estudiante), hallamos un efecto significativo en el autocultivo, $\chi^{2}(2, N=667)$ $=11.96, p<.01, \phi^{\prime}$ de Cramer $=.134$. Los análisis a posteriori revelaron que los que están laboralmente activos au- 
tocultivan significativamente más $(p<.017)$ y con un tamaño del efecto pequeño, $d=0.31$, que los estudiantes.

\section{Autocultivo y patrones de consumo}

La frecuencia de consumo reciente de cannabis está relacionada significativamente con el autocultivo, $\chi^{2}(4, N=758)$ $=48.46, p<.001, \phi^{\prime}$ de Cramer $=.25$. Concretamente, los que consumieron con mayor frecuencia (a diario) en el último mes (.46) autocultivan significativamente más $(p<.005)$ que los que no consumieron ninguno, $d=0.49$, o los que lo hicieron con menor frecuencia, es decir, entre 1 y 3 días $(d=$ $0.51)$ y entre 4 y 9 días $(d=0.40)$. Esto y, a pesar de la autoproducción que resulta insuficiente, lleva a que los que autocultivan hayan tenido que poner excusas sobre su gasto de dinero en una proporción (.37) significativamente mayor, aunque con un tamaño del efecto pequeño, $\chi^{2}(1, N=761)=$ $5.28, p<.05, d=0.17$, que los que no cultivan (.29). Asimismo, el autocultivo (.65 vs. .42, para autocultivo y no autocultivo, respectivamente) se asocia, significativamente y con un tamaño del efecto pequeño, con el consumo de otras drogas (i.e., cocaína, éxtasis), $\chi^{2}(1, N=746)=33.79, p<$ $.001, d=0.44$.

Los individuos que cultivan plantas de cannabis (.42) consumen en mayor medida, y con un tamaño del efecto pequeño, en solitario, $\chi^{2}(1, N=759)=17.38, p<.001, d=$ 0.31 , que los que no la cultivan (.28).

Los individuos (.63) que autocultivan cannabis se relacionan significativamente más, $\chi^{2}(1, N=759)=9.95, p<$ .01 , y con un tamaño del efecto pequeño, $d=0.23$, con otros consumidores que los que no cultivan la planta de cannabis (.51).

La preocupación por el dinero invertido en el consumo de cannabis es significativamente mayor, $\chi^{2}(1, N=761)=$ $14.78, p<.001$, aunque con un tamaño del efecto pequeño, $d$ $=0.28$, entre los que autocultivan (.49) que entre aquellos que no lo hacen (.35).

Los consumidores con autocultivo (.72) han vendido drogas ilegales significativamente en mayor medida, $\chi^{2}(1, N$ $=754)=96.96, p<.001, \mathrm{y}$ con un tamaño del efecto grande, $d=0.77$, que los que no autocultivan (.34). Asimismo, ambos estratos poblaciones difieren entre sí en el motivo principal para la venta ilegal (consumir gratis, ganar dinero y otros), $\chi^{2}(2, N=248)=12.47, p<.01, \phi^{\prime}$ de Cramer $=.22$. Los contrastes a posteriori $(p<.017)$ evidenciaron que los que autocultivan la venden para ganar dinero (.57), mientras que los que no autocultivan (.66) lo hacen para consumir gratis, y con un tamaño del efecto pequeño, $d=0.47$.

En relación al lugar más habitual de adquisición de cannabis (lugar público vs. vivienda del proveedor), los resultados no sugieren un patrón de adquisición diferente mediado por el autocultivo, $\chi^{2}(1, N=473)=0.07, n s, d=-0.02$, siendo, en general, la vivienda del proveedor de cannabis (.69) el lugar habitual de adquisición, $\chi^{2}(1, N=473)=66.24, p<$ $.001, \delta=0.97$.
El consumo de cannabis en el ámbito recreativo se asocia con otras drogas legales (i.e., alcohol y tabaco), en más de la mitad de la población de consumidores, $\chi^{2}(1, N=723)=$ $76.38, p<.001$, y con un tamaño del efecto grande, $\delta=0.84$. Sin embargo, no se hallaron diferencias significativas $\chi^{2}(1, N$ $=723)=0.00, n s, d=0.00$, en este patrón de policonsumo entre los que autocultivan (.51) y los que no autocultivan $(.50)$.

\section{Autocultivo y riesgo de consumo problemático (CAST)}

En términos de riesgo de consumo problemático, más de la mitad (.59) de los consumidores habituales de cannabis son clasificados, $\chi^{2}(1, N=744)=27.10, p<.001, \delta=0.30$, como de alto riesgo ( $p d \geq 7$ en CAST; Spilka, Janssen, y Legleye, 2013). Sucintamente, los consumidores que autocultivan son clasificados como de riesgo de consumo problemático (.78) significativamente más, $\chi^{2}(1, N=744)=49.81, p<$ .001 , y con un tamaño del efecto moderado, $d=0.54$, que los que no autocultivan (.51). En la misma línea, los resultados de la comparación de medias de los grupos con y sin autocultivo en el riesgo de consumo problemático (CAST) mostraron que el grupo de autocultivo $(M=9.44)$ presenta un riesgo de consumo problemático significativamente mayor, $t(513)=6.66, p<.001, \mathrm{y}$ de un tamaño del efecto moderado, $d=0.51$, que los del grupo de no-autocultivo $(M=$ 7.06).

\section{Autocultivo y dependencia del cannabis (SDS)}

Más de la mitad (.56) de los consumidores habituales de cannabis cumplen criterios diagnósticos de dependencia, $\chi^{2}(1, N=718)=8.91, p<.01, \delta=0.28$. Por su parte, los consumidores con autocultivo (.64) son diagnosticados $(p d \geq$ 3 en SDS; Swift, Copeland, y Hall, 1998) más frecuentemente de dependencia del cannabis, $\chi^{2}(1, N=718)=10.08, p<$ .01 , y con un tamaño del efecto pequeño, $d=0.19$, que los que no autocultivan (.52). Asimismo, el contraste de medias inter-grupos puso de manifiesto que los que autocultivan $(M$ $=4.20)$ presentan un nivel de dependencia psicológica significativamente mayor, $t(716)=4.05, p<.001, \mathrm{y}$ de un tamaño del efecto pequeño, $d=0.32$, que los que no practican el autocultivo $(M=3.23)$. A su vez, la población adulta de consumidores $(M=3.55)$ es psicológicamente dependiente del cannabis (valor de prueba $=3$, esto es, el punto de corte de dependencia psicológica), $t(717)=4.84, p<.001$, con un tamaño del efecto pequeño, $d=0.18$.

\section{Autocultivo y síntomas y trastornos asociados (FTND, AUDIT, CPQ)}

Los consumidores habituales que autocultivan y los que no autocultivan presentan por un igual dependencia al tabaco (FTND), $t(415)=0.43, n s, d=0.04$. Por su parte, la población de consumidores habituales de cannabis $(M=3.17)$ 
no es dependiente del tabaco (valor de prueba $=6$ ), $t(568)=$ $-28.56, p<.001$, con un tamaño del efecto más que grande, $d$ $=-1.20$. El estudio de casos de dependencia de la nicotina, muestra que no hay una asociación entre dependencia de la nicotina y el autocultivo, $\chi^{2}(1, N=569)=1.19, n s, d=0.09$.

Los resultados de los análisis del consumo de riesgo de alcohol (AUDIT) para la variable de agrupamiento autocultivo muestran un nivel de riesgo similar para ambos grupos, $t(340)=-0.36, n s, d=-0.03$. Ahora bien, la población de consumidores de cannabis $(M=9.92)$ es una población de consumo de riesgo de alcohol (valor de prueba $=8), t(696)=$ -8.57, $p<.001$, y con un tamaño del efecto pequeño, $d=$ 0.32 . Finalmente, el estudio de casos evidencia igual número de casos entre consumidores con autocultivo y sin autocultivo, $\chi^{2}(1, N=697)=0.03, n s, d=0.01$.
Los resultados de la comparación del grupo de autocultivo y de no-autocultivo en la problemática psicosocial (i.e., física, psicológica y social) asociada al consumo habitual de cannabis (CPQ), mostró diferencias entre ambos grupos, $F(3,742)=8.59, p<.001, \eta_{\mathrm{p}}{ }^{2}=.034,1-\beta=.994$. Por su parte, los efectos univariados (ver Tabla 1 ) concretaron que dichas diferencias se circunscribían a las consecuencias sociales (p.e., problemas policiales, más relación con amigos que también fuman) así como a las consecuencias psicológicas (p.e., sentirse deprimido, falta de motivación) que eran mayores para el grupo de autocultivo. Finalmente, la población de consumidores habituales de cannabis está en la región de normalidad (datos tomados para el cómputo de Copeland et al., 2005), tanto en la problemática psicosocial en su conjunto, como en cada una de las dimensiones que la componen, física, psicológica y social.

Tabla 1. Efectos univariados en la problemática psicosocial relacionada con el uso habitual del cannabis para el factor autocultivo (sí vs. no).

\begin{tabular}{llllll}
\hline Escalas & $F$ & $p$ & $\eta_{\mathrm{p}}^{2}$ & $M_{\mathrm{ga}}$ & $M_{\mathrm{gn}-\mathrm{a}}$ \\
\hline Física & 0.33 & .566 & .000 & 3.08 & 3.17 \\
Psicológica & 4.17 & .042 & .006 & 2.09 & 1.81 \\
Social & 15.73 & .000 & .021 & 1.83 & .088 \\
\hline
\end{tabular}

Nota. $g l(1,744) ; M_{\mathrm{ga}}=$ Media del grupo que autocultiva cannabis; $M_{\mathrm{gn}-\mathrm{a}}=$ Media del grupo que no autocultiva cannabis.

El autocultivo (.29), frente al consumo sin autocultivo (.21), está ligado, en mayor medida, $\chi^{2}(1, N=761)=6.12, p$ $<.05$, y con un tamaño del efecto pequeño, $d=0.18$, a la pérdida de peso.

Por último, hallamos que los consumidores con autocultivo manifiestan padecer con mayor frecuencia $(M=2.82)$ problemas de memoria, dificultades para resolver tareas mentales complejas (pensamiento) o deterioro de la capacidad de juicio, $t(751)=3.93, p<.001$, que los que no autocultivan $(M=2.46)$, con un tamaño del efecto pequeño, $d=$ 0.30 .

\section{Discusión}

En línea con hallazgos previos (Gamella y Jiménez, 2003), el autocultivo no es un comportamiento altamente distintivo de los consumidores $(p<.5)$, aunque sí significativo $(p>.05)$, adquiriendo los productos para el autocultivo generalmente en Grow Shops. Este dato viene a confirmar que las Grow Shops desempeñan un papel central en la expansión del autocultivo y, por extensión, de la cultura cannábica (Calafat, Juan et al., 2000; Isorna, 2013).

Los resultados ponen de manifiesto que el patrón de consumo es radicalmente diferente entre los que autocultivan y los que no lo hacen. En concreto, los que autocultivan se perfilan, en contraste con los que no autocultivan, por ser mayormente varones, laboralmente activos y con menor formación académica; excusar más el gasto vinculado al consumo; mostrar una mayor preocupación por el gasto económico; presentar una mayor tasa de venta de drogas con el objeto de ganar dinero; consumir más en soledad; relacionarse con una red de consumidores; policonsumo; por consu- mir más frecuentemente (a diario); presentar una probabilidad mayor riesgo de consumo problemático; y cumplir con mayor frecuencia los criterios para el diagnóstico de dependencia del cannabis.

Por lo que se refiere a la relación del género con el autocultivo, la literatura advierte que la tendencia diferencial en el consumo habitual de esta sustancia tiende a desaparecer, dado que se está observando una convergencia entre los patrones de consumo y uso de cannabis entre hombres y mujeres (Nebot, Giménez, Ariza, y Tomás, 2006; Observatorio Español sobre Drogas, 2013). Consecuentemente, se espera una tendencia similar en el autocultivo de cannabis para el propio consumo. Sin embargo, en las restantes variables se espera continuidad.

Las variables que perfilan al autocultivador como laboralmente activo y con menor formación académica forman, a nuestro entender, una unidad. Así, está suficientemente demostrada la relación entre rendimiento académico y consumo de cannabis, bien porque el consumo interfiere negativamente en el funcionamiento escolar por medio del síndrome amotivacional, del daño cognitivo generado o del entorno social frecuentado (Lynskey y Hall, 2000; Verweij, Huizink, Agrawai, Martin, y Lynskey, 2013), bien porque el bajo rendimiento académico conlleva a que se expongan a comportamientos de riesgo de desviación social, incluido el consumo de cannabis (Hall y Solowij, 1998).

Hipotéticamente, la justificación del autocultivo descansa fundamentalmente en razones económicas (i.e., satisfacción del consumo, ganancia con la venta), o bien del propio producto consumido (i.e., calidad, pureza). A este respecto, encontramos que el autocultivo se vincula con la preocupación y excusación del gasto producido por el consumo, así como 
con la venta ilegal con el fin de obtener beneficios económicos. Estos resultados, que engranan cultivo doméstico con connotaciones económicas (Decorte, 2011; Nguyen y Bouchard, 2010; Potter, 2010), suponen un paso más en una hipotética escala de gravedad dado que se asocia a connotaciones económicas, dejando un margen menor justificativo para la otra causa argüida para el cultivo: la garantía de calidad y pureza del producto (Decorte, 2010).

Contrariamente a la hipótesis de que el control paterno inhibe el consumo de drogas y, más aún, el cultivo (Velleman, Templeton, y Copello, 2005), los datos sostienen que el control paterno es independiente del autocultivo (téngase presente que se limita a hijos mayores de edad). Por tanto, los padres o bien toleran el autocultivo o, lo que parece menos probable, no son conscientes del mismo.

Socialmente, el autocultivo se relaciona con un patrón de mayor consumo (i.e., en soledad, diario, en redes), la participación en redes de consumidores (Calafat, Fernández, et al., 2000; de la Haye, Green, Kennedy, Pollard, y Tucker, 2013) y policonsumo (Font-Mayolas, Gras, y Planes, 2006). Este entramado social y de consumo es facilitador de la permanencia en su consumo (Parrott, Morinan, Moss, y Scholey, 2004), así como de la recaída (Dishion y Owen, 2002; Yao, Ciesla, Mazurek, y Spear, 2012), al tiempo que obstaculiza el tratamiento (Bobes y Calafat, 2000).

Clínicamente y como consecuencia de todo lo anterior, los que practican autocultivo presentan una probabilidad mayor de riesgo de consumo problemático; y cumplen con mayor frecuencia los criterios para el diagnóstico de dependencia del cannabis. Estos resultados otorgan una alta consistencia interna a la secuencia de resultados anteriores ya que todos los indicadores advertían de mayor riesgo y gravedad clínica para los que practican autocultivo.

En suma, el perfil del consumidor habitual de cannabis que autocultiva es indicativo de un nivel superior de escalada (Kandel y Yamaguchi, 1985) en el consumo de drogas y, por inferencia, un factor de riesgo para otros problemas de salud mental (Hall y Degenhardt, 2009; Kuepper et al., 2011; Parolaro, 2010) y de uso de otras drogas ilegales (Mayet, Legleye, Falissard, y Chau, 2012; Moss, Chen, y Yi, 2014; Swift et al., 2012).

El autocultivo implica un mayor riesgo de consumo problemático y una mayor probabilidad de desarrollo de un patrón de abuso de cannabis que descansa mayormente en sintomatología clínica física (p.e., pérdida de peso), cognitiva (p.e., problemas de memoria, dificultades para resolver tareas mentales complejas, deterioro de la capacidad de juicio), y afectación de las actividades sociales (y, por deducción, laborales) y recreativas. Investigación futura debe dirigirse a observar la consistencia de la relación entre pérdida de peso y autocultivo y, de ser persistente, identificar las causas porque se ha dado por hecho (American Psychiatric Association, 2014) y se ha encontrado una explicación biológica para la relación contraria, la ganancia de peso, asociada a la estimulación del apetito (Martín-Banderas, Durán-Lobato, Holgado, Álvarez-Fuentes, y Fernández-Arévalo, 2013). Adicionalmente hay que añadir que, como consumidores habituales de cannabis, los que autocultivan también asocian el consumo al alcohol, es decir, a otras sustancias (Gervilla, Cajal, Roca, y Palmer, 2010), al tiempo que estos comportamientos de consumo también se relacionan con ingresos penitenciarios (García-Jiménez, Godoy-Fernández, Llor-Esteban, y Ruiz-Hernández, 2014).

En conclusión, el autocultivo, la consecuente venta del producto con el objetivo prioritario de ganar dinero, y la problemática psicosocial derivada del consumo habitual de cannabis conlleva una trayectoria en la inadaptación social (Arce, Fariña, y Vázquez, 2011; Arce, Seijo, Fariña, y Mohamed-Mohand, 2010); escalada en la gravedad del consumo (Kandel y Yamaguchi, 1985); una especificación de mayor gravedad clínica, de consumo y psicosocial (American Psychiatric Association, 2014); y a una menor competencia cognitiva (Arce, Fariña, y Novo, 2014).

Estos resultados traen aparejado que la prevención de la escalada en el consumo y el tratamiento de consumidores habituales de cannabis que autocultivan o no, han de ser diferentes porque los riesgos así lo son, además de los síntomas y trastornos clínicos asociados.

Finalmente es preciso tener en mente las limitaciones del diseño planteado en este estudio que afectan a la generalización de los resultados. Primera, los resultados se basan en autoinformes de los propios consumidores con el consabido sesgo de respuesta inherente a este tipo de poblaciones (p.e., disimulación). Segunda, es posible incurrir en un sesgo de muestreo utilizando el método bola de nieve pues, por sus características, el subgrupo de población seleccionado puede ser poco representativo. Tercera, los resultados se obtuvieron con población adulta por lo que no tienen por qué ser generalizables a la población de menores. Cuarta, las clasificaciones y diagnósticos se fundamentan en instrumentación psicométrica por lo que no son realmente diagnósticos clínicos, sino impresiones diagnósticas.

\section{Referencias}

American Psychiatric Association. (2014). Manual diagnóstico y estadístico de los trastornos mentales (DSM-V). Madrid: Panamericana.

Arce, R., Fariña, F., Carballal, A., y Novo, M. (2006). Evaluación del daño moral en accidentes de tráfico: Desarrollo y validación de un protocolo para la detección de la simulación. Psicothema, 18, 278-283.

Arce, R., Fariña, F., y Novo, M. (2014). Competencia cognitiva en penados primarios y reincidentes: Implicaciones para la reeducación. Anales de Psicología, 30(1), 259-266.
Arce, R., Fariña, F., y Vázquez, M. J. (2011). Grado de competencia social y comportamientos antisociales delictivos y no delictivos en menores. Revista Latinoamericana de Psicología, 43, 473-486.

Arce, R., Seijo, D., Fariña, F., y Mohamed-Mohand, L. (2010). Comportamiento antisocial en menores: Riesgo social y trayectoria natural de desarrollo. Revista Mexicana de Psicología, 27, 127-142.

Asociación Médica Mundial. (2013, Octubre). Declaración de Helsinki. Principios éticos para las investigaciones médicas en seres humanos. 64a. Asamblea Ge- 
neral, Fortaleza, Brasil. Recuperado de http://www.wma.net/es/30publications/10policies/b3/

Becoña, E., Martínez, U., Calafat, A., Fernández-Hermida, J. R., Juan, M., Sumnall, H., Mendes, F., y Gabrhelík, R. (2013). Parental permissiveness, control, and affect and drug use among adolescents. Psicothema, 25, 292-298. doi:10.7334/psicothema2012.294

Bobes, J., y Calafat, A. (2000). De la neurobiología a la psicosociología del uso-abuso del cannabis. Adicciones, 12(Supl. 2), 7-17.

Bovasso, G. B. (2001). Cannabis abuse as a risk factor for depressive symptoms. American Journal of Psychiatry, 158, 2033-2037. doi:10.1176/appi.ajp.158.12.2033

Calafat, A., Fernández, C., Becoña, E., Gil, E., Juan, M., y Torres, M. A. (2000). Consumo y consumidores de cannabis en la vida recreativa. Adicciones, 12(Supl. 2), 197-230.

Calafat, A., Juan, M., Becoña, E., Fernández, C., Gil, E., y Llopis, J. J. (2000). Estrategias y organización de la cultura pro-cannabis. Adicciones, 12(Supl. 2), 231-273.

Coffey, C., Lynskey, M., Wolfe, R., y Patton G. C. (2000). Initiation and progression of cannabis use in a population-based Australian adolescent longitudinal study. Addiction, 95, 1679-1690. doi:10.1046/j.13600443.2000.951116798.x

Cohen, J. (1988). Statistical power analysis for the behavioral sciences (2nd ed.). Hillsdale, NJ: LEA.

Copeland, J., Gilmour, S., Gates, P., y Swift, W. (2005). The cannabis problems questionnaire: Factor structure, reliability, and validity. Drug and Alcobol Dependence, 80, 313-319. doi:10.1016/j.drugalcdep.2005.04.009

Davis, G. P., Compton, M. T., Wang, S., Levin, F. R., y Blanco, C. (2013). Association between cannabis use, psychosis, and schizotypal personality disorder: Findings from the National Epidemiologic Survey on Alcohol and Related Conditions. Schizophrenia Research, 151, 197-202. doi:10.1016/j.schres.2013.10.018

Decorte, T. (2010). Small scale domestic cannabis cultivation: An anonymous web survey among 659 cannabis cultivators in Belgium. Contemporary Drug Problems, 37, 341-370

Decorte, T., Potter, G. R., y Bouchard, M. (2011). The globalization of (domestic) cannabis cultivation. En T. Decorte, G. R. Potter, y M. Bouchard (Eds.), World, wide, weed: Global trends in cannabis cultivation and its control (pp. 1-22). Farnham: Ashgate.

de la Haye, K., Green, H. D., Kennedy, D. P., Pollard, M. S., y Tucker, J. S. (2013). Selection and influence mechanisms associated with marijuana initiation and use in adolescent friendship networks. Journal of Research on Adolescence, 23, 474-486. doi:10.1111/jora.12018

Di Forti, M., Sallis, H., Allegri, F., Trotta, A., Ferraro, L., Stilo, S.,... Murray, R. M. (2014). Daily use, especially of high-potency cannabis, drives the earlier onset of psychosis in cannabis users. Schizopbrenia Bulletin. Advance online publication. doi:10.1093/schbul/sbt181

Dishion, T. J., y Owen, L. D. (2002). A longitudinal analysis of friendships and substance use: Bidirectional influence from adolescence to adulthood. Developmental Psychology, 38, 480-491. doi:10.1037/00121649.38.4.480

Font-Mayolas, S., Gras, M. E., y Planes, M. (2006). Análisis del patrón de consumo de cánnabis en estudiantes universitarios. Adicciones, 18, 337344.

Gamella, J. F., y Jiménez, M. L. (2003). El consumo prolongado de cánnabis: Pautas, tendencias y consecuencias. Madrid: Fundación de Ayuda contra la Drogadicción FAD

García-Jiménez, J. J., Godoy-Fernández, C., Llor-Esteban, B., y RuizHernández, J. A. (2014). Differential profile in partner aggressors: Prison vs. mandatory community intervention programs. The European Journal of Psychology Applied to Legal Context, 6, 69-77.

Gervilla, E., Cajal, B., Roca, J., y Palmer, A. (2010). Modelling alcohol consumption during adolescence using zero inflated negative binomial and decision trees. The European Journal of Psychology Applied to Legal Context, 2, 145-159.

Gervilla, E., y Palmer, A. (2010). Prediction of cannabis and cocaine use in adolescence using decision trees and logistic regression. The European Journal of Psychology Applied to Legal Context, 2, 19-35.

González-Saiz, F., de las Cuevas, C., Barrio, G., y Domingo-Salvany, A. (2008). Versión española consensuada de la Severity of Dependence
Scale (SDS). Medicina Clínica, 131, 797-798. doi:10.1016/S00257753(08)75509-X

Gossop, M., Darke, S., Griffiths, P., Hando, J., Powis, B., Hall, W., y Strang, J. (1995). The Severity of Dependence Scale (SDS): Psychometric properties of the SDS in English and Australian samples of heroin, cocaine and amphetamine users. Addiction, 90, 607-614. doi:10.1046/j.13600443.1995.9056072.x

Hall, W., y Degenhardt, L. (2009). Adverse health effects of non-medical cannabis use. The Lancet, 374, 1383-1391. doi:10.1016/S01406736(09)61037-0

Hall, W., y Solowij, N. (1998). Adverse effects of cannabis. The Lancet, 352, 1611-1616.

Heatherton, T. F., Kozlowski, L. T., Frecker, R. C., y Fagerström, K. O. (1991). The Fagerström Test for Nicotine Dependence: A revision of the Fagerström Tolerance Questionnaire. British Journal of Addictions, 86, 1119-1127. doi:10.1111/j.1360-0443.1991.tb01879.x

Hedges, L. V., y Olkin, I. (1985). Statistical methods for meta-analysis. San Diego, CA: Academic Press.

International Narcotics Control Board. (2011). Report of the International Narcotics Control Board for 2011. New York: United Nations. Recuperado de http://www.unodc.org/documents/southasia/reports/2011_INCB_A NNUAL_REPORT_english_PDF.pdf

Isorna, M. (2013). La evidencia científica en la prevención del consumo de cannabis. En M. Isorna y D. Saavedra (Eds.), Prevención de drogodependencias y otras conductas adictivas (pp. 257-287). Madrid: Pirámide.

Isorna, M., Fernández-Ríos, L., y Souto, A. (2010). Treatment of drug addiction and psychopathology: A field study. The European Journal of Psychology Applied to Legal Context, 2, 3-18.

Kandel, D. B., y Yamaguchi, K. (1985). Developmental patterns of the use of legal, illegal, and medically prescribed psychotropic drugs from adolescence to young adulthood. En C. L. Jones y R. J. Battjes (Eds.), Etiology of drug abuse (pp.193-235). Rockville, MD: Nacional Institute on Drug Abuse.

Kraemer, H. C., y Andrews, G. (1982). A non-parametric technique for meta-analysis effect size calculation. Psychological Bulletin, 91, 404-412.

Kuepper, R., van Os, J., Lieb, R., Wittchen, H. U., Hofler, M., y Henquet, C. (2011). Continued cannabis use and risk of incidence and persistence of psychotic symptoms: 10 year follow-up cohort study. British Medical Journal, 342, d738. doi:10.1136/bmj.d738

Legleve, S., Karila, L., Beck, F., y Reynaud, M. (2007). Validation of the CAST, a general population Cannabis Abuse Screening Test. Journal of Substance Use, 12, 233-242. doi:10.1080/14659890701476532

Ley Orgánica 15/1999 de 13 de diciembre de Protección de Datos de Carácter Personal. (1999). BOE, 298, 43088-43099. Recuperado de http://www.boe.es/boe/dias/1999/12/14/pdfs/A43088-43099.pdf

Lynskey, M., y Hall, W. (2000). The effects of adolescent cannabis use on educational attainment: A review. Addiction, 95, 1621-1630. doi:10.1046/j.1360-0443.2000.951116213.x

Maldonado, R., Valverde, O., y Berrendero, F. (2006). Involvement of the endocannabinoid system in drug addiction. Trends in Neurosciences, 29, 225-232. doi:10.1016/j.tins.2006.01.008

Martín del Moral, M., y Lorenzo, P. (1998). Conceptos fundamentales en drogodependencias. En P. Lorenzo, J. M. Ladero, J. C. Leza, y I. Lizasoain (Eds.), Drogodependencias: Farmacologia, patologia, psicologia y legislación (pp. 3-21). Madrid: Editorial Médica Panamericana.

Martín-Banderas, L., Durán-Lobato, M., Holgado, M. Á., Álvarez-Fuentes, J., y Fernández-Arévalo, M. (2013). Interés terapéutico de cannabinoides: Análisis bibliométrico en Pubmed, Scopus y Web of Science. Revista Iberoamericana de Psicología y Salud, 4, 17-30.

Mayet, A., Legleye, S., Falissard, B., y Chau, N. (2012). Cannabis use stages as predictors of subsequent initiation with other illicit drugs among French adolescents: Use of a multi-state model. Addictive Behaviors, 37, 160-166. doi:10.1016/j.addbeh.2011.09.012

Moss, H. B., Chen, C. M., y Yi, H. Y. (2014). Early adolescent patterns of alcohol, cigarettes, and marijuana polysubstance use and young adult substance use outcomes in a nationally representative sample. Drug and Alcobol Dependence, 136, 51-62. doi:10.1016/j.drugalcdep.2013.12.011

Nebot, M., Giménez, E., Ariza, C., y Tomás, Z. (2006). Tendencias en el consumo de tabaco, alcohol y cannabis en los adolescentes de Barcelona entre 1987 y 2004. Medicina Clinica, 126, 159. doi:10.1157/13084030 
Nguyen, H., y Bouchard, M. (2010). Patterns of youth participation in cannabis cultivation. Journal of Drug Issues, 40, 264-293. doi:10.1177/002204261004000202

Observatorio Español sobre las Drogas. (2011). Situación y tendencias de los problemas de drogas en España. Madrid: Ministerio de Sanidad, Servicios Sociales e Igualdad. Recuperado de http://www.pnsd.msc.es/Categoria2/observa/pdf/oed2011.pdf

Observatorio Español sobre Drogas. (2013). Encuesta domiciliaria sobre alcohol y drogas en población general en España (EDADES) 2011/2012. Madrid: Ministerio de Sanidad, Servicios Sociales e Igualdad. Recuperado de http://www.pnsd.msc.es/Categoria2/observa/pdf/EDADES2011.pdf

Observatorio Español sobre Drogas. (2014). Encuesta estatal sobre uso de drogas en enseñanzas secundarias (ESTUDES) 2012/2013, España. Madrid: Ministerio de Sanidad, Servicios Sociales e Igualdad. Recuperado de http://www.pnsd.msc.es/Categoria2/observa/pdf/PresentESTUDES 2012_2013.pdf

Organización Mundial de la Salud. (1992). CIE-10: Clasificación de los trastornos mentales y del comportamiento. Madrid: Meditor.

Palmer, A., Borrás, C., Pérez-Pareja, J., Sesé, A., y Vilariño, M. (2013). Are patients with chronic pain and fibromyalgia correctly classified by MMPI-2 validity scales and indexes? The European Journal of Psychology Applied to Legal Context, 5, 123-129. doi:10.5093/ ejpalc2013a1

Parolaro, D. (2010). Consumo de cánnabis de los adolescentes y esquizofrenia: Evidencias epidemiológicas y experimentales. Adicciones, 22, 185190.

Parrott, A., Morinan, A., Moss, M., y Scholey, A. (2004). Understanding drugs and behaviour. Chichester, UK: John Wiley and Sons.

Patton, G. C., Coffey, C., Carlin, J. B., Degenhardt, L., Lynskey, M., y Hall, W. (2002). Cannabis use and mental health in young people: Cohort study. British Medical Journal, 325, 1195-1198. doi:10.1136/bmj.325.7374.1195

Potter, D. J. (2014). A review of the cultivation and processing of cannabis (Cannabis sativa L.) for production of prescription medicines in the UK. Drug Test Analysis, 6, 31-38. doi:10.1002/dta.153

Potter, D. J., Clark, P., y Brown, M. B. (2008). Potency of $\Delta^{9}-$ THC and other cannabinoids in cannabis in England in 2005: Implications for psychoactivity and pharmacology. Journal of Forensic Sciences, 53, 90-94. doi:10.1111/j.1556-4029.2007.00603.x

Potter, G. R. (2010). Weed, need and greed: A study of domestic cannabis cultivation. Londres: Free Association Books.

Rubio-Valladolid, G., Bermejo-Vicedo, J., Caballero Sánchez-Serrano, M. C., y Santo-Domingo Carrasco, J. (1998). Validation of the Alcohol Use
Disorders Identification Test (AUDIT) in primary care. Revista Clinica Española, 198, 11-14.

Saunders, J. B., Aasland, O. G., Babor, T. F., de la Fuente, J. R., y Grant, M. (1993). Development of the Alcohol Use Disorders Identification Test (AUDIT): WHO collaborative project on early detection of persons with harmful alcohol consumption-II. Addiction, 88, 791-804. doi:10.1111/j.1360-0443.1993.tb02093.x

Spilka, S., Janssen, E., y Legleye, S. (2013). Detection of problem cannabis use: The Cannabis Abuse Screening Test (CAST). Paris: Observatoire Français des Drogues et des Toxicomanies.

Swift, W., Coffey, C., Degenhardt, L., Carlin, J. B., Romaniuk, H., y Patton, G. C. (2012). Cannabis and progression to other substance use in young adults: Findings from a 13-year prospective population-based study. Journal of Epidemiology \& Community Health, 66, e26. doi:10.1136/jech.2010.129056

Swift, W., Copeland, J., y Hall, W. (1998). Choosing a diagnostic cut-off for cannabis dependence. Addiction, 93, 1681-1692.

United Nations Office on Drugs and Crime. (2013). World drug report. Vienna, Austria. Retrived from http://www.unodc.org/documents/dataand-analysis/WDR2012/WDR_2012_web_small.pdf

Velleman, R. D. B., Templeton, L. J., y Copello, A. G. (2005). The role of the family in preventing and intervening with substance use and misuse: A comprehensive review of family interventions, with a focus on young people. Drug and Alcohol Review, 24, 93-109.

Verdejo-García, A. (2011). Efectos neuropsicológicos del consumo de cannabis. Trastornos Adictivos, 13, 97-101.

Verweij, K. J. H., Huizink, A. C., Agrawai, A., Martin, N. G., y Lynskey, M. T. (2013). Is the relationship between early-onset cannabis use and educational attainment causal or due to common liability? Drug and Alcohol Dependence, 133, 580-586. doi:10.1016/j.drugalcdep.2013.07.034

von Sydow, K., Lieb, R., Pfister, H., Höfler, M., y Wittchen, H. U. (2002). What predicts incident use of cannabis and progression to abuse and dependence? A 4-year prospective examination of risk factors in a community sample of adolescents and young adults. Drug and Alcohol Dependence, 68, 49-64. doi:10.1016/S0376-8716(02)00102-3

Yao, P., Ciesla, J. R., Mazurek, K. D., y Spear, S. F. (2012) Peer relations scale for adolescents treated for substance use disorder: A factor analytic presentation. Substance Abuse Treatment, Prevention, and Policy, 7, 29. doi:10.1186/1747-597X-7-29

(Artículo recibido: 27-01-2015; revisado: 05-04-2015; aceptado: 14-04-2015) 